EPiC Series in Engineering
Volume 2, 2018, Pages 56-66
SUMO 2018- Simulating Autonomous
and Intermodal Transport Systems

\title{
Coupling traffic and driving simulation: Taking advantage of SUMO and SILAB together
}

\author{
Mirko Barthauer ${ }^{1}$ and Alexander Hafner ${ }^{2}$ \\ 1 Technische Universität Braunschweig, Institute of Transportation and Urban Engineering, \\ Hermann-Blenk-Straße 42, 38108 Braunschweig, Germany \\ 2 Technische Universität Braunschweig, Institute of Automotive Engineering, \\ Hans-Sommer-Straße 4, 38106 Braunschweig, Germany
}

\begin{abstract}
Driving simulators provide a safe testing environment for reactions of human drivers in traffic. Traffic simulations traditionally focus on deducing infrastructure efficiency measures. Both types of simulation model similar aspects but differ in scale and detail. They mostly target different research domains, too, like psychologists, automotive or traffic engineers. Current traffic studies tend to simplify driving assistance systems a lot. As those get developed further, interdisciplinary collaboration may help to model their impact on the traffic system. A short literature overview of simulation couplings in this field, their applications and challenges is given. In this work, a coupling mechanism is being developed to run the driving simulation SILAB and the microscopic traffic simulation SUMO in parallel. Components include a mutual traffic participant exchange, traffic light states transfer, and an automatic road network converter. Here, the technical concept of a closed loop as well as the planned application are presented. The human-driven car from SILAB is placed into $S U M O$ and makes the other vehicles react, and vice versa: $S U M O$-controlled vehicles act as surrounding traffic in SILAB. Firstly, this facilitates the driving simulation of urban road networks with many random traffic participants. Secondly, microscopic traffic simulation may profit from the insights gained from the test persons driving in SILAB.
\end{abstract}

\section{Introduction}

Traffic in general can be seen as people and goods moving from one place to another. Depending on the field of research, there are different levels of detail traffic is modeled. Each level splits the traffic system into more subsystems: The so-called macroscopic level uses aggregated measures like time-dependent traffic volumes to model the movements. Looking closer at it, individual entities like vehicles, people and traffic control elements start to emerge in microscopic level. On one hand, traffic engineers use both detail levels frequently for traffic quality studies, but also assess ITS (intelligent transportation systems) applications with their help. On the other hand, automotive engineers and psychologists rather stick to the submicroscopic level: Vehicles can move independently of lanes and interactions between human beings, vehicle components and the environment can be studied explicitly. 
With more and more Advanced Driver Assistance Systems (ADAS) being developed, it becomes important to know whether these have a non-negligeable effect on the traffic system as a whole. Automotive engineers use a driving simulator to study the effect on the driver itself and the environment nearby. Traffic engineers need to scale the ADAS effect to a vehicle fleet and to different road network conditions in a microscopic traffic simulation. To put it short, one depends on the other to implement its own study: Traffic engineers need an ADAS model and automotive engineers can feed back the result in their ADAS development.

Up to now, a common simulation environment for both has been rather an exception. In this work, a coupling of the driving simulation SILAB and the microscopic traffic simulation SUMO shows how ADAS can be evaluated in a more integrated way. Firstly, previous simulation couplings are reviewed in terms of expected effects, challenges and their (technical) solutions. Among them, there are some $S U M O$ examples which have been explored carefully. Afterwards, differences and similarities between $S U M O$ and SILAB are highlighted before going into details about the coupling concept and its planned application.

\section{Literature review}

Over the last decades, some concepts for coupling driving simulation and microscopic traffic simulation have been realised. Sometimes, coupling is also referred to as co-simulation [15]. In general, these terms define a parallel execution of both simulations: Using comparable road networks, information about the traffic participants is exchanged regularly. The human driver in the driving simulator experiences traffic reacting on his "ego-vehicle". Information about other components like the traffic signals may be shared, too. After conducting the driving simulator study, test persons' behaviour can be evaluated with respect to the driving style. In fact, the latter could be used to improve existing behavioural models inside the simulations.

It seems quite reasonable that the very first concepts for simulation couplings started to appear only after enough computing power for both simulation types had become available. At the national French transport research institute then called INRETS (IFSTTAR today), Espié and Auberlet date the idea of using a common simulation called ARCHISIM/SIM ${ }^{2}$ to the early 1990s [4]. From a computer science perspective, Klein et al. show how both driving and traffic simulations can cooperate by means of a High Level Architecture (HLA) [8].

In recent years, more researchers coupled various traffic and driving simulation products. Almost everybody came up with a new combination: Punzo and Ciuffo [12] as well as Nguyen That and Casas [10] used AIMSUN and SCANeR, Olstam connected internally-developed simulations [11], Hou et al. used an in-house driving simulator and Paramics [7], and Schiller et al. chose Virtual Test Drive with SUMO [13]. This might be due to the following:

- Relatively few (serious) driving simulator installations around the world but quite some competing driving and traffic simulation products

- Deep integration of driving simulator hardware and software

- Costs for installation and user training

Nonetheless, some important lessons can be retained from previous works. Firstly, the road networks of both simulations have to match closely for a bidirectional exchange of movements. Automatic converters between open file formats like RoadXML [10] or OpenDrive [13] and proprietary traffic simulation file formats guarantee for consistent geometries. Secondly, driving simulations need to be executed in real time thus recalculated more frequently than usual traffic 
Table 1: Couplings of SUMO with driving simulations

\begin{tabular}{r|c|l}
\hline Authors & Year & Driving simulation \\
\hline Laquai [9] & 2014 & Virtools-based in-house development \\
Schiller et al. [13] & 2015 & Virtual Test Drive \\
Semrau et al. [14] & 2016 & Virtual Test Drive \\
Aramrattana [1] & 2016 & VTI in-house development \\
Döbler et al. [3] & 2016 & In-house development \\
\hline
\end{tabular}

simulations. Hou et al. interpolate movements [7] while others create a moving zone around the "ego-vehicle" where all traffic participants are controlled by the driving simulation (see [10], [13]). This leads to how the data exchange should be organized: Either an independent controller [15] [7] or a plugin of the driving simulation [13] synchronize the simulations sending network communication messages caught by the respective API (application programming interface). Current implementations do not necessarily follow the HLA approach exactly.

There have been more couplings $S U M O$ was involved as shown in table 1. In general, SUMO interacts with the driving simulation through the Traffic Control Interface (TraCI), e.g. to trigger a time step calculation. Sometimes the coupling logic is bundled in a driving simulator plugin [13] or given as an independent program [14]. The latter may be more future-proof because all general coupling issues like data exchange and synchronisation are not linked to a specific simulation. Aramrattana's system [1] includes the network communication simulation OMNET + + while the others stick to SUMO and their driving simulation.

Up to now, most of the simulation couplings focused on the technical concept but feature case studies including a specific road network type. Rural roads and motorways (see [12], [13], [14]) prevail over urban scenarios with traffic lights and intersections (see [7]). Research questions do not differ significantly from other driving simulator studies and cover

- Driving behaviour in general

- Human-machine-interface design

- ADAS design

- Road design

Olstam explains major reasons why traffic and driving simulations are used together: The surrounding traffic accounts for a lot of the simulator realism and affects the study validity indirectly. Test persons behave differently when they just play a game [11]. Traffic simulation provides agents driving according to their own plans and capabilities. This contributes to a more realistic driving simulation which otherwise often relies on single predefined vehicle trajectories. Olstam sees a trade-off between exposing each test person to exactly defined situations to be tested and having more realistic surrounding traffic [11].

Up to here, the mentioned coupling concepts serve automotive engineers for enhancing driving simulator realism. Traffic simulation experts can analyse the driving simulator study results with respect to their own objectives. Only then they can integrate the observed phenomena into their methods and scale to a fleet in the traffic simulation. In this paper, the proper coupling is referred to as "online" due to its synchronised execution while the transfer of results is considered "offline". In 2006, Aronsson [2] calibrated speed on urban streets using driving simulator data. Hoffmann [6] modeled driving under ADAS conditions by fitting speed distributions to SILAB driving simulation and real-world tests. In theory, ADAS development may 
also be influenced by a backward transfer of traffic simulation results to the driving simulator environment. However, no preceding application has been found in this context.

To conclude the literature review, some important points should be highlighted. The previous works indicate a need for a coherent road network definition and a synchronisation concept between the simulations involved. Both simulation couplings as defined here (online/offline) belong together and have their place. In constrast to rural roads and motorways, simulation coupling has not been applied much to urban scenarios with signalized intersections yet.

\section{Current state}

The driving simulator hardware contributes heavily to the study results. Test persons experience realistic driving through visual, sound and haptic feedback. That is why both the driving simulator and the driving simulation software $S I L A B$ are presented with the coupling in mind. Where necessary, the SILAB concept is compared to the SUMO approach.

\subsection{Driving simulator hardware}

In early 2015, Technische Universität Braunschweig inaugurated the center for automotive and mobility research "Niedersächsisches Forschungszentrum Fahrzeugtechnik" (NFF). It comprises the "Dynamic Vehicle Road Simulator" (DVRS) pictured in figure 1 with which human-machineinterface (HMI), chassis and steering systems and the driver's subjective impression are studied. Test persons are seated in an original Volkswagen Golf VII GTD facing curved monitors for a 180 degree round view of the virtual environment. The car has been modified before installing it as a mockup: Only the front part was retained and the combustion engine has been replaced by an electric one. Working air-conditioning keeps test persons from getting dizzy. They can use the cockpit buttons and the multi-functional steering wheel like when driving on real roads. Additionally, several monitors serve as speedometer, displays and side-view mirrors.

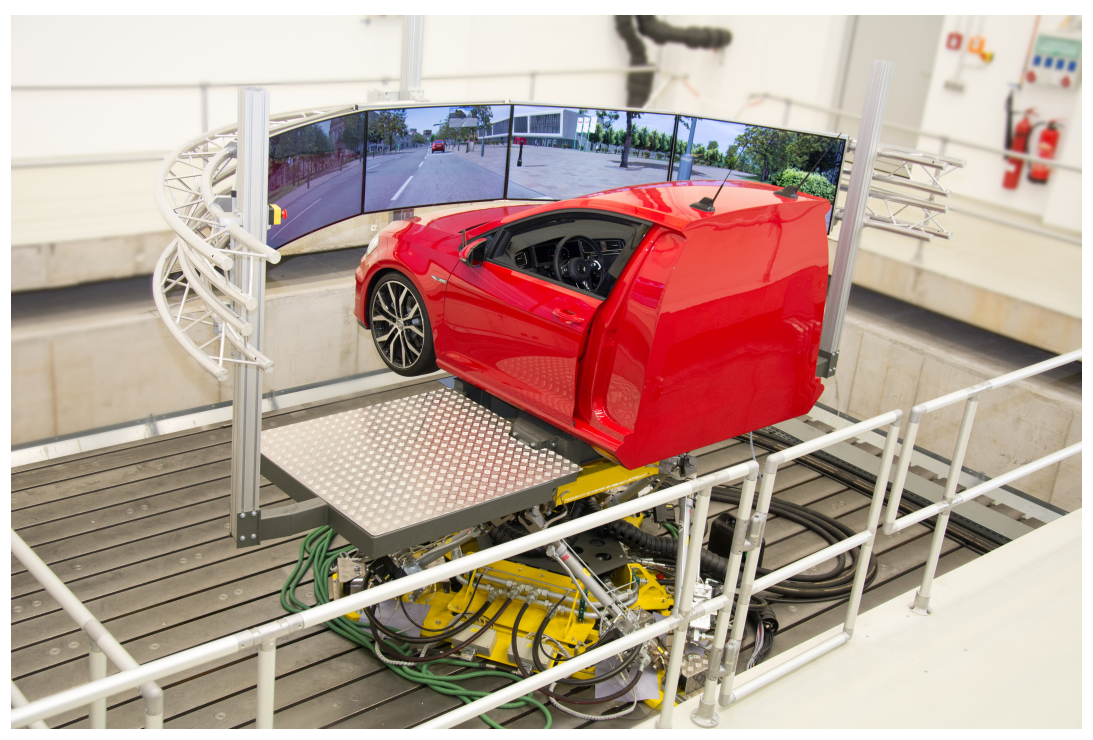

Figure 1: NFF Dynamic Vehicle Road Simulator 
The mockup sits on a hydraulic hexapod which can make it move at maximum $1 \mathrm{~g}$ to let the passengers feel the acceleration. All in all, the equipment facilitates testing and optimizing ADAS with respect to the driver. Calculation tasks are distributed in the associated computer network and coordinated by the driving simulation software. [5]

\subsection{Driving simulator software}

The DVRS uses the driving simulation software SILAB [16] for all basic tasks. The software manages inputs and outputs, standard vehicle dynamics and surrounding traffic. It provides interfaces for custom sensors, vehicle dynamics, ADAS and HMI. The states of several objects can be observed during the simulation, e.g. the test driver's speed and position. Before driving in a scenario, the road geometry, other traffic participants and traffic management have to be defined. At the beginning of the project, it was considered an option to switch to another driving simulation like Virtual Test Drive which had already proven to work with SUMO (see table 1). Up to now, the DVRS has been configured for use with SILAB and other users of teh DVRS depend on it. Though the potential implications on other studies and the overall DVRS organisation made that $S I L A B$ remains in place.

\subsubsection{Road network definition}

SILAB uses a multi-level approach to build the road network from smaller modules. These atomar subnetworks can be of type "Course" for a sequence of curves sharing the same cross section or of type "Area2" for intersections and lane extensions of any kind. They can be merged with each other at predefined border points. Due to its dynamic network feature, instances of the road modules can be repeated ad infinitum - an orthogonal city network could be built with a single intersection module. When the test driver is leaving the current module, the following is chosen depending on his behaviour. This creates geometric inconsistencies as the same space is occupied by different modules over time. The feature can be disabled by setting

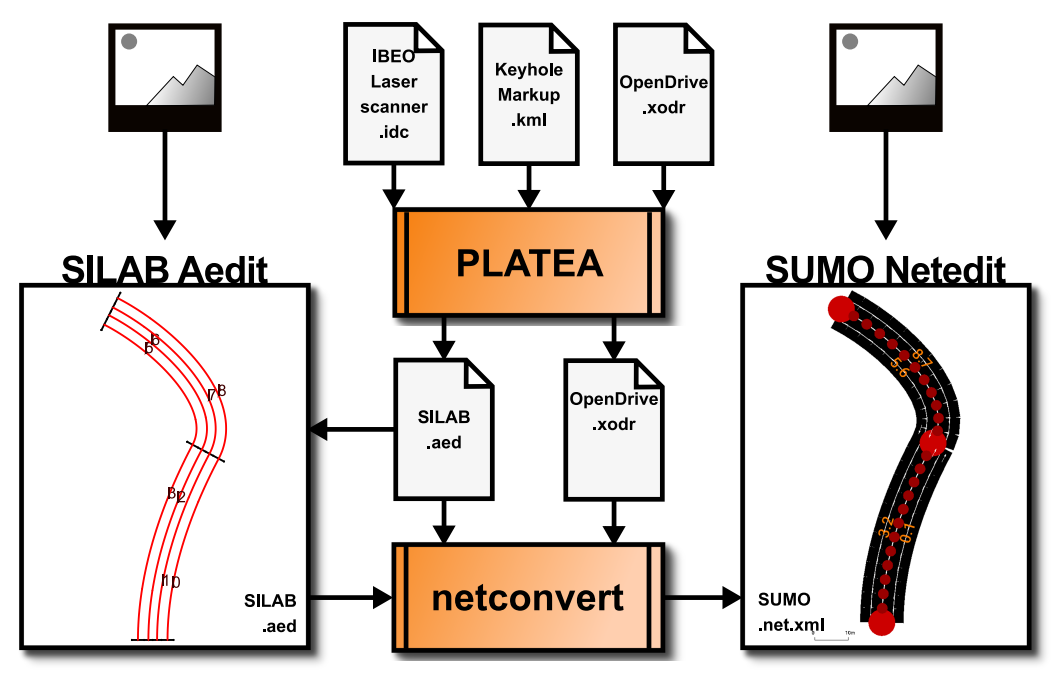

Figure 2: Road network conversion process 
static rules for the next module. Every module is defined in human-readable text files (.aed file ending) using a custom language. At the moment of writing, "Area2" modules can be created in the supplied graphic editor SILABAedit using background images but cannot be converted automatically from other types of road descriptions by default.

At module level, the road is built out of two major components: "Cross sections" define the number, width and direction of lanes at a specific chainage. Proper "Lane" elements connect outgoing and incoming lane ports of two cross sections with each other. Lanes can be merged by ending at the same lane port. Depending on the angle in between, they are represented by straight lines or Bézier curves. Additional elements are placed on the lanes to model stop lines, pedestrian crossings and traffic lights. The small "Area2" module example included in figure 2 shows three cross sections with two lanes per direction connected among each other.

All in all, SILAB road networks share some concepts with SUMO but not everything. Each simulation run in $S U M O$ is associated with exactly one road network given as a graph of directed edges and junctions. Edges represent one or more lanes of the same carriageway between two junctions. Inside the junction borders, inner edges show which incoming lane is linked to which outgoing lane. This way, stop lines, pedestrian crossings and traffic lights are always placed at the end of a lane. In any case, lane geometry is built from linear segments which are extruded by a constant width. Curves are approximated by many short segments. The geometry points are defined in a 2,5D orthonal coordinate system. Over the years, automatic converters for well-known formats like OpenStreetMap and OpenDrive into SUMO XML network definitions have been developed.

Some facts stand out: The dynamic road network approach of SILAB cannot be used together with $S U M O$. As junctions are not modeled explicitly in SILAB, they need to be identified in the conversion process. Edges are not modeled, either. Finally, lane coordinates need to be adapted carefully to fit Bézier curves, stop lines and junction shapes.

\subsubsection{Adaptation and extendibility}

SILAB provides the Dynamic Processing Unit (DPU) mechanism to read and to control simulation components at every time step. A DPU can be understood as an additional feature packed into a dynamic linked library. It has a number of predefined input and output variables and can be instantiated through the simulation configuration file. There, the output of one DPU can be directed to another as an input. There is a set of DPUs shipped with the software to access specific parts of the simulation, say the traffic lights. During the simulation, the linked input variables will be updated with the value of the previous time step. Thus, a custom DPU can be configured to read almost any value in the simulation and overwrite some through its input/output configuration. Valuable and available simulation attributes for the "online" coupling are included in table 2. ADAS systems of the ego-vehicle can be modeled by a DPU, too.

Table 2: Valuable SILAB simulation attributes

\begin{tabular}{r|c|l}
\hline Table 2: Valuable SILAB simulation attributes \\
Atribute & \multicolumn{2}{|c}{ Access } \\
& Read & Control \\
\hline Signal control & Yes & Yes \\
Vehicle dynamics of the surrounding traffic & Yes & Untested* \\
Vehicle dynamics of the ego-vehicle & Yes & No \\
\hline
\end{tabular}

* Feature not available yet 
In theory, control over specific simulated items could be divided between the coupled simulations. As long as not every item can be controlled externally in both simulations, this concept cannot be fully implemented. On the $S U M O$ side, the Traffic Control Interface (TraCI) grants read and control access to the simulated signal control and vehicle dynamics. Hence $S U M O$ can take over the signal control in SILAB, but cannot transfer its vehicles in the driving simulation. This feature has not been available yet at the moment of writing but exists as stated by the software producer.

\subsubsection{Temporal terms}

Driving simulations have to run at realtime. Otherwise, test persons are irritated by the delayed feedback due to a too low execution rate. Every extension of the simulation should take care not to brake this rule. Using an execution rate of $60 \mathrm{~Hz}$ equals a time step of ca. $17 \mathrm{~ms}$ in constrast to usual $S U M O$ time steps in the range $[0.1 ; 1.0] \mathrm{s}$.

\section{Coupling SUMO and SILAB}

As already pointed out in section 2, coupling driving and traffic simulations creates some challenges. The differences between $S U M O$ and $S I L A B$ in modeling road networks and the synchronisation issue have been studied in section 3.2. The following paragraphs detail how the encountered challenges can be overcome.

\subsection{Road network}

Due to different level of detail, road network descriptions will always differ a bit between traffic and driving simulations. Nonetheless, the objective should be to find analogous road networks without map matching issues of the vehicle positions. Vehicles should change lanes and turn smoothly when controlled by $S U M O$. It seems obvious to automate the network creation process for that. Hafner et al. have already developed a versatile semi-automatic tool chain called PLATEA to convert data of multiple sources to SILAB road network modules [5]. However, intersections have not been addressed for now as they result much more complicated than curve segments. Consequently, two distinct options can be considered for generating analogous networks for $S I L A B$ and $S U M O$ :

1. Derive the $S U M O$ network directly from SILAB: Draw a road network in the graphical editor SILABAedit (equivalent of Netedit) using background images. Convert the resulting .aed file to a $S U M O$ network within an enhanced netconvert version. The result can be checked using the same background images in Netedit.

2. Generate SILAB and SUMO networks from a common data source using the tools PLATEA and netconvert. Available data sources include IBEO laser scanner data, Keyhole Markup Language and OpenDrive files.

Figure 2 illustates the approaches described above. Currently, the enhanced netconvert handles only curve segments successfully. Parallel SILAB lanes going in the same direction are grouped to $S U M O$ edges and connect to the subsequent edge. Conversion of intersections, traffic lights and ulterior details is still under development. It is still a long road until dynamic road networks (see section 3.2.1) might work in the simulation coupling. An option could be to let SUMO simulate exactly one "Area2" road network and load a new one when the test person approaches 


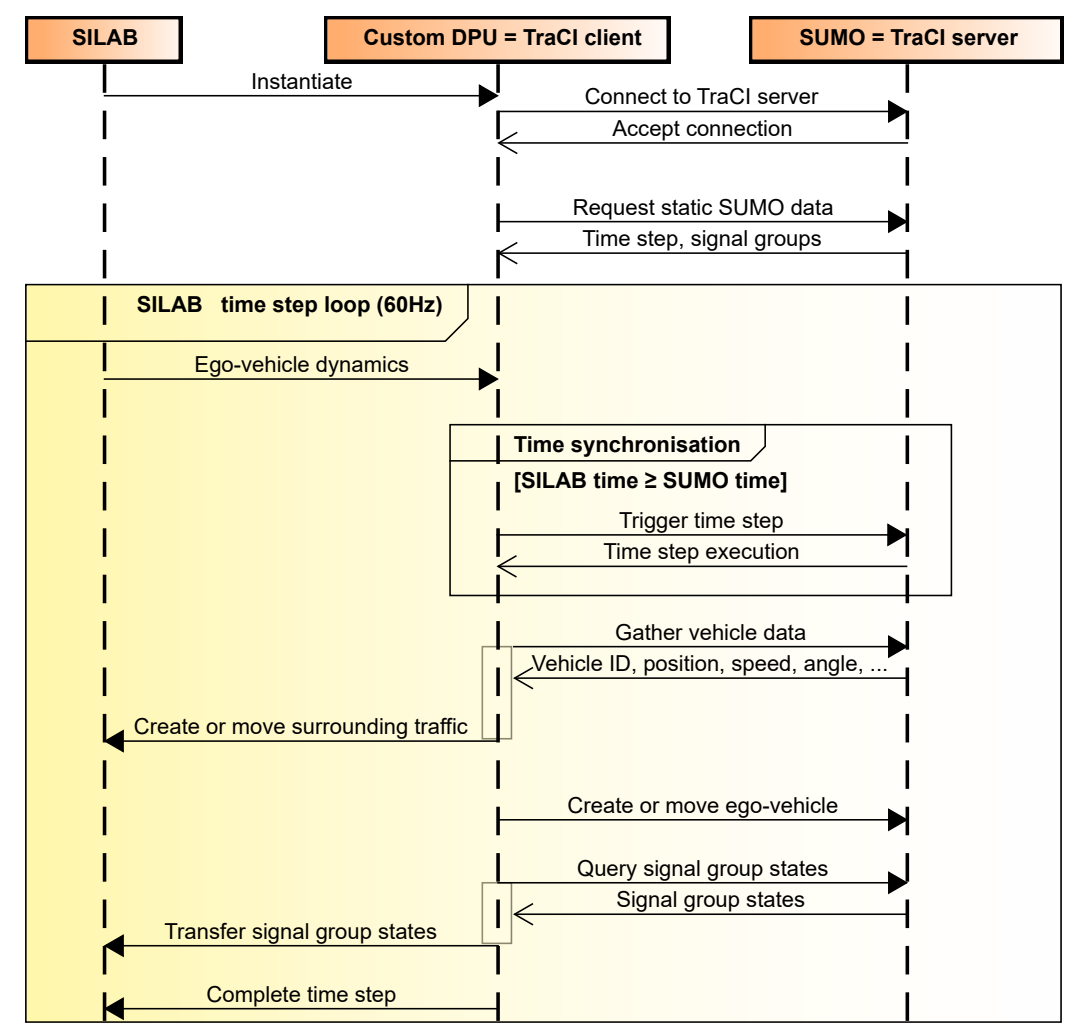

Figure 3: Sequence diagram of the "online" coupling execution

the border. Then, boundary conditions with respect to coordinates and time should be looked at very closely.

\subsubsection{Synchronisation}

The synchronisation is the second essential part for a working "online" coupling of SILAB and $S U M O$. It has to be ensured that both simulations are aware of the same traffic participants for any simulated time. This boils down to a frequent information exchange. Like stated in section 2 , concepts of varying complexity exist for how the coupled simulations communicate during execution. The following approach is tied to the software and therefore cannot be transferred easily to other applications. On the other hand, it can be implemented quite fast.

All synchronisation logic has been grouped in custom SILAB DPUs which interact with the simulations themselves. The DPU instance communicates with SILAB via its input and output variables. Additionally, it registers itself with $S U M O$ via TraCI calls. This way, the DPU instance has access to the needed attributes in both simulations. Figure 3 shows the kind of exchanged messages in chronological order and how often they are sent. In first place, the simulations are initialised and connect to each other. Consequently, static information which does not change over the whole simulation is gathered once. As $S U M O$ time steps may last longer than $S I L A B$ ones, the DPU has to record them and trigger a time step calculation only when SILAB is late. Then, the "ego-vehicle" copy in the traffic simulation is updated. The 
surrounding traffic in SILAB shall be updated, too. As stated previously, this step has not been realised yet. Finally, the traffic light states are requested, translated and written to DPU output variables. They will be effective starting from the next SILAB time step.

\subsection{Verification}

At this point, the "online" coupling is still awaiting completion because of the missing control over vehicles in SILAB. Meanwhile, the coupling concept presented previously is verified in a small example network. The verification is considered an antecedent step of the validation to ensure the planned features work as expected. It is intended to perform a real validation once the system has been completed.

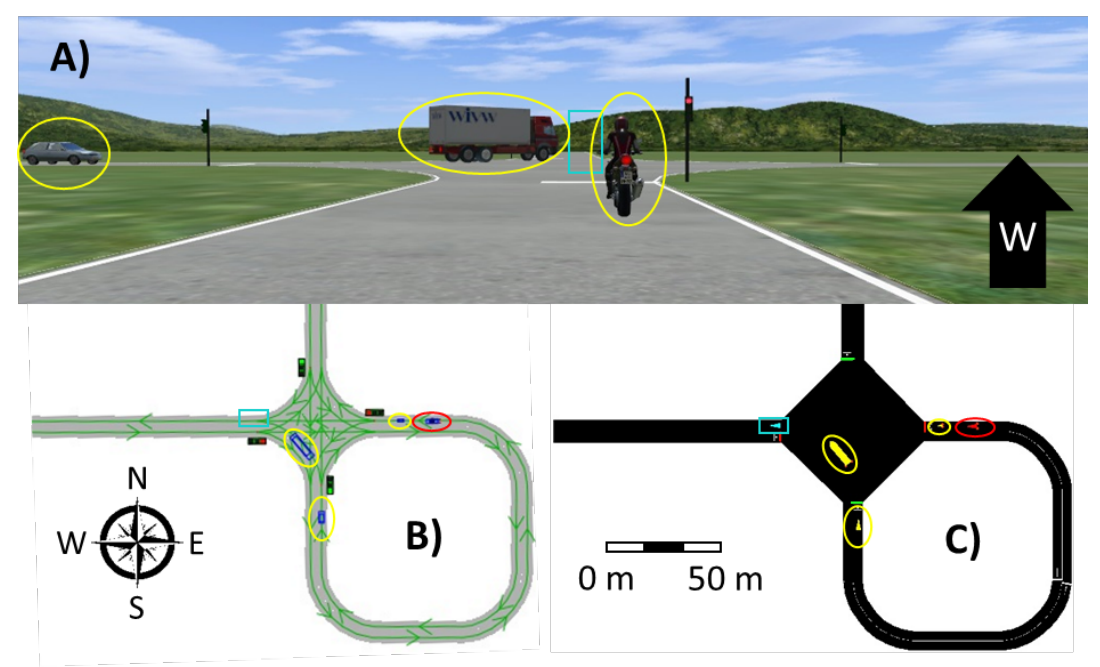

Figure 4: Views on the same situation (A: SILAB driver view, B: SILAB bird view, C: SUMO)

A small road network (see figure 4) consisting of a four-leg signalised intersection has been designed using the respective graphical network editors. Two approaches have been linked with each other through a loop. Multiple views of the same scene in both simulations show traffic participants on their way. In this case, we only test vehicles transferred to $S U M O$. For example, the truck turns left at the intersection as his signal shows green. This informal visual verification has to be seconded by an advanced evaluation once all features of the coupling concept have been implemented.

\subsection{Planned application}

The simulation coupling under development shall be used on a long-term base for different kinds of studies. Two options are being prepared for now:

1. ADAS evaluation: Assess the individual and system effects of a speed advising system in urban environment which takes signal control into account.

2. Human driver model: Derive a more detailed model of human driving and propagate it to the whole fleet in the traffic simulation. 


\section{Conclusion}

The coupling concepts involving traffic and driving simulation provide automotive and traffic engineers with new complementary features. Human interaction and the effect of ADAS can be evaluated both at driver and traffic system level: ADAS and human behaviour can be better integrated into traffic simulation while surrounding traffic can react more naturally to test persons driving. Preconditions for a succesful coupling of $S I L A B$ and $S U M O$ include analogous road networks, synchronisation of traffic participants and signal control during simulation execution. From a technical perspective, this goal is tackled by extending $S I L A B$ in a custom DPU and converting the road network automatically. We expect to complete its implementation soon. Before applying the coupling to ADAS and human behaviour studies, an advanced verification and validation will take place. An advanced version of the simulation coupling might be able to deal with dynamic road networks. That way, the study design could benefit from a bigger degree of freedom.

\section{Acknowledgement}
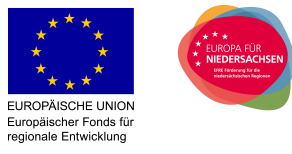

This paper is based on the research project "Simulationskopplung für einen sicheren und nachhaltigen Verkehr" funded by the European Regional Development Fund.

\section{References}

[1] Maytheewat Aramrattana. Modelling and Simulation for Evaluation of Cooperative Intelligent Transport System Functions. PhD thesis, Högskolan i Halmstad, 2016. Accessed 02.03.2017 at http://hh.diva-portal.org/smash/get/diva2:968642/FULLTEXT01.pdf.

[2] Karin F.M. Aronsson. Speed characteristics or urban streets based on driver behaviour studies and simulation. PhD thesis, Kungliga Tekniska högskolan (KTH), 2006.

[3] Holger Döbler, Stefan Dietzel, Johannes Eising, Felix Fischer, Jan Flaig, Olaf Florian, Christoph Fritze, Karmel Indych, Markus Montenegro, Sebastian Müller, Markus Schneider, Nicole Vieregg, Andreas Wegge, Florian Koller, Olaf Menzel, and Björn Scheuermann. PARCOURS: A SUMOIntegrated 3D Driving Simulator for Behavioral Studies. In SUMO2016 - Traffic, Mobility, and Logistics, Berlin, May 2016.

[4] Stephane Espié and Jean-Michel Auberlet. Joint use of driving simulation and traffic simulation for the study of road infrastructure equipments. In Joint International Conference on Computing and Decision Making in Civil and Building Engineering, June 2006.

[5] Alexander Hafner, Adrian Sonka, Roman Henze, and Ferit Küçükay. Integration of the real measurement data into the DVRS. In chassis.tech plus 2016, München, June 2016.

[6] Silja Hoffmann. Mikroskopische Modellierung und Bewertung von verkehrssicherheitskritischen Situationen : am Beispiel kommunikationsbasierter Fahrerwarnungen auf Autobahnen. PhD thesis, Technische Universität München, München, 2014.

[7] Yunfei Hou, Yunjie Zhao, Kevin F. Hulme, Shan Huang, Yaqin Yang, Adel W. Sadek, and Chunming Qiao. An integrated traffic-driving simulation framework: Design, implementation, and validation. Transportation research Part C, 45:138-153, 2014.

[8] Ulrich Klein, Thomas Schulze, and Steffen Straßburger. Traffic simulation based on the High Level Architecture. In Proceedings of the 1998 Winter Simulation Conference, 1998. 
[9] Florian Ulrich Laquai. Anticipation Assistance For Drivers. PhD thesis, Technische Universität München, 2014. Accessed 02.03.2017 at https://www.mmk.ei.tum.de/fileadmin/w00bqn/www/ Dissertationen/Laquai_komprimiert.pdf.

[10] Thomas Nguyen That and Jordi Casas. An integrated framework combining a traffic simulator and a driving simulator. Procedia - Social and Behavioral Sciences, 20:648-655, 2011.

[11] Johan Olstam. Simulation of Surrounding Vehicles in Driving Simulators. PhD thesis, Linköpings Universitet, 2009.

[12] Vincenzo Punzo and Biagio Ciuffo. Integration of driving and traffic simulation: Issues and first solutions. IEEE transactions on intelligent transportation systems, 12(2):354-364, 2011.

[13] Manuel Schiller, Marius Dupuis, Daneil Krajzewicz, Andreas Kern, and Alois Knoll. MultiResolution Traffic Simulation for Large Scale High Fidelity Evaluation of VANET Applications. In SUMO2015 - Intermodal Simulation for Intermodal Transport, May 2015.

[14] Marc Semrau, Jakob Erdmann, Bernhard Friedrich, and René Waldmann. Simulation framework for testing ADAS in Chinese traffic situations. In SUMO2016 - Traffic, Mobility, and Logistics, Berlin, May 2016.

[15] Marc Semrau, Jakob Erdmann, Jens Rieken, and Bernhard Friedrich. Modelling and calibrating situation adaptive lane changing and merging behavior on chinese elevated roads. In SUMO2017 - Towards Simulation for Autonomous Mobility, pages 15-28, Berlin, May 2017.

[16] WIVW GmbH. Driving Simulation and SILAB. Web page. Accessed 21.02.2018 at https: //wivw.de/en/silab. 